\title{
Cyclic heptapeptide microcystin biosynthesis requires the glutamate racemase gene
}

\author{
Tomoyasu Nishizawa, ${ }^{1,3}$ Munehiko Asayama ${ }^{1}$ and Makoto Shirai ${ }^{1,2}$
}

Author for correspondence: Makoto Shirai. Tel: +81 29888 8652. Fax: +81 298888653. e-mail: shirai@ipc.ibaraki.ac.jp

1,2 Laboratory of Molecular Genetics, School of Agriculture ${ }^{1}$, and Gene Research Center2, Ibaraki University, Ami, Ibaraki 300-0393, Japan

3 The United Graduate School, Tokyo University of Agriculture and Technology, Fuchu, Tokyo 183-8509, Japan

\begin{abstract}
It was demonstrated previously that the operon consisting of the nonribosomal peptide synthetase (NRPS) gene coupled with the polyketide synthase (PKS) gene involved in cyclic heptapeptide microcystin synthesis includes two different $D$-amino acid synthetase genes, an epimerization domain at the $3^{\prime}$ end of module 2, and the racemase gene mcyF. To determine the role of mcyF in microcystin synthesis, gene-disruption and complementation analyses were carried out. Insertional mutagenesis in the mcyF gene, generated by homologous recombination, abolished only microcystin synthesis, but did not influence cell growth. Furthermore, McyF supported D-Glu-independent growth of a strain of Escherichia coli defective in D-Glu synthesis. It is concluded that $m c y F$ is the glutamic acid racemase gene involved in the synthesis of D-Glu residues in the microcystin molecule. This is the first report of the racemase in prokaryotic NRPS.
\end{abstract}

Keywords: amino acid racemase, cyanobacteria, Microcystis, peptide synthetase gene, microcystin biosynthesis

\section{INTRODUCTION}

The cyanobacteria (blue-green algae) are Gram-negative and are exceptionally large photosynthetic prokaryotes. These bacteria proliferate in eutrophic marine and freshwater habitats, resulting in the formation of water blooms. Toxic cyanobacterial water blooms are found worldwide in eutrophic lakes, ponds and dams (Carmichael, 1994; Watanabe et al., 1996). The major cause of toxicity is hepatotoxic microcystin, and the toxicity of microcystins is due to the inhibition of protein phosphatases 1 and 2A (MacKintosh et al., 1990). Microcystins cause a variety of human illnesses and death in indigenous and domestic animals. Recently, the deaths of more than 50 haemodialysis patients in Caruaru, Brazil, were attributed to exposure to microcystin in the dialysis water (Jochimsen et al., 1998). The general structure of microcystin (MCYST-XZ) is cyclo (-D-Ala-X-D-MeAsp-Z-Adda-D-Glu-Mdha-), in which ' $\mathrm{X}$ ' and ' $\mathrm{Z}$ ' are variable $\mathrm{L}$-amino acids, $\mathrm{Mdha}$ is $\mathrm{N}$ methyldehydroalanine, D-MeAsp is D-erythro- $\beta$-methylaspartic acid, and Adda is 3-amino-9-methoxy-10-

Abbreviations: Adda, 3-amino-9-methoxy-10-phenyl-2,6,8-trimethyldeca-4,6-dienoic acid; MCYST, microcystin; Mdha, $\mathrm{N}$-methyldehydroalanine; D-MeAsp, D-erythro- $\beta$-methylaspartic acid; NRPS, non-ribosomal peptide synthetase; PKS, polyketide synthase; UDP-MurNAc-L-Ala-D-Glu, UDP- $N$-acetylmuramoyl-L-alanyl-D-glutamate. phenyl-2,6,8-trimethyl-deca-4,6-dienoic acid (Fig. 1). More than 60 structural variants of microcystins have been found (Sivonen \& Jones, 1999).

Peptides including microcystins that may contain nonprotein amino acids are synthesized non-ribosomally by a large multifunctional enzyme, utilizing a thio-template mechanism called non-ribosomal peptide synthetase (NRPS) (Stachelhaus \& Marahiel, 1995; Marahiel, 1997). We have cloned the microcystin synthetase genes (mcy $A,-B,-C,-D,-E,-F$ and $-G$ ) encoding seven NRPS modules and polyketide synthases (PKSs) for synthesis of the Adda molecule (Nishizawa et al., 2000). There are three NRPSs (McyA, McyB and McyC), two NRPS-PKS hybrids (McyE and McyG) and one PKS (McyD). Furthermore, in addition to the epimerase gene of module 2, a racemase gene for $\mathrm{D}$-amino acid synthesis was also observed. The microcystin molecule has three D-amino acids: D-Ala, D-MeAsp and D-Glu. The same D-amino acids incorporated in non-ribosomally synthesized peptides by known prokaryotic NRPSs are synthesized by epimerization domains. Only one epimerization domain was found at the carboxy-terminal end of the thiolation domain in module 2 of McyA. Since this module is responsible for activation and incorporation of Ala, this epimerase domain must be for D-Ala synthesis.

Many D-amino acid racemase genes for peptidoglycan 


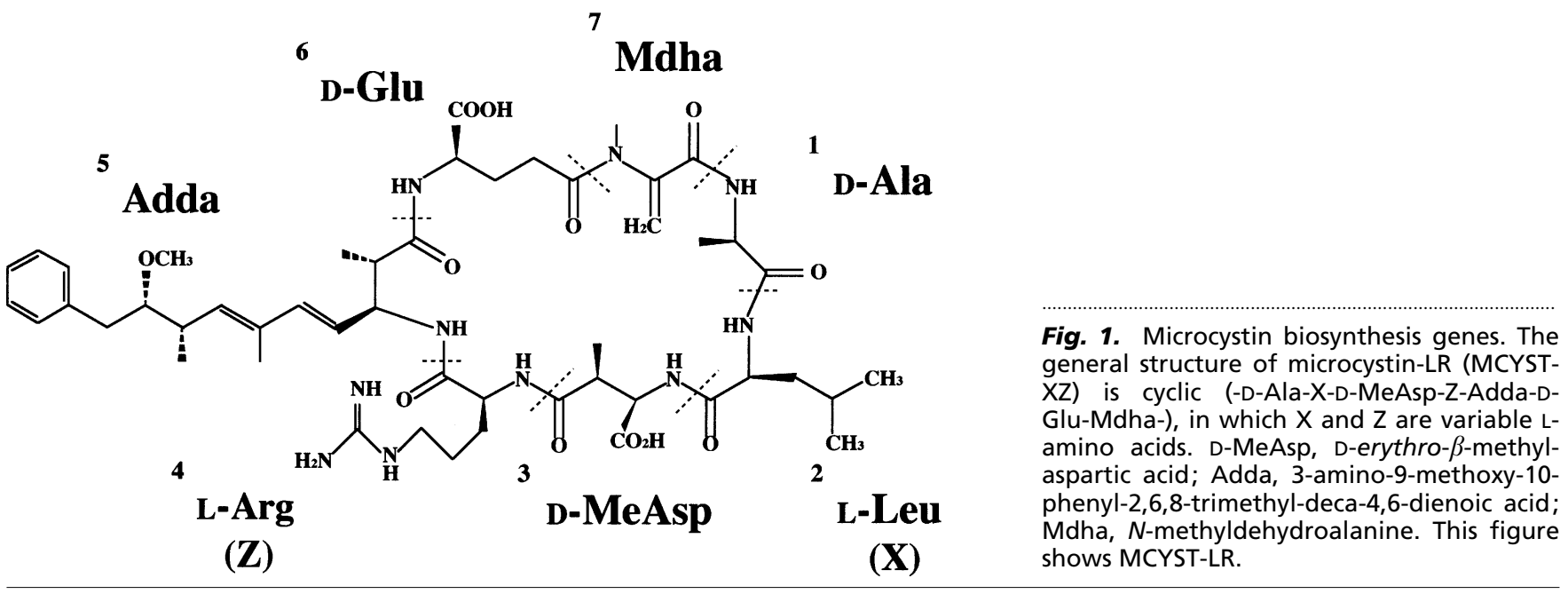

synthesis have been reported in eubacteria (Liu et al., 1997; Malathi et al., 1999; Pucci et al., 1995). However, no racemase genes have been reported in prokaryotic NRPS. The alanine racemase genes responsible for synthesis of cyclosporin A and HC-toxin were identified in fungal NRPS (Cheng \& Walton, 2000; Hoffmann et al., 1994). In this study, we showed that the $m c y F$ gene encodes the glutamate racemase involved in microcystin synthesis.

\section{METHODS}

Bacterial strains and growth conditions. Microcystis aeruginosa K-139 (which produces 7-desmethyl-MCYST-LR and 3,7-didesmethyl-MCYST-LR) and Microcystis sp. S-70 (which produces MCYST-LR, -RR and -YR), both of which were isolated from Lake Kasumigaura, Ibaraki (Harada et al., 1991; Sakamoto et al., 1993), were used in this study. Microcystis strains were grown under continuous illumination (2000 lx) at $30^{\circ} \mathrm{C}$ in CB medium (Shirai et al., 1991). Escherichia coli DH5 $\alpha$ MCR (Cosmo Bio.) and E. coli JM109 (ToYoBo) were used as hosts for recombinant plasmids and were grown at $37^{\circ} \mathrm{C}$ in $2 \times$ TY broth and $2 \times$ TY agarose. $E$. coli strain WM335 (leu pro trp his arg thy A met lac gal rpsL $h s d M$ hsdR murI gltS) was used for a complementation test (Lugtenberg et al., 1973). Antibiotics were used for recombinants, which were grown at final concentrations of $75 \mu \mathrm{g}$ ampicillin $\mathrm{ml}^{-1}$ and $25 \mu \mathrm{g}$ chloramphenicol $\mathrm{ml}^{-1}$.

DNA manipulation, Southern blotting and sequencing. Total DNA of Microcystis cells was isolated by the method described previously (Sakamoto et al., 1993), and DNA manipulations were performed as described by Sambrook et al. (1989). Digested cyanobacterial DNA was separated in $0.8 \%$ agarose gels and transferred onto Hybond-NX membranes. DNA fragments (as probes) were labelled using an ECL random prime labelling kit (Amersham Pharmacia Biotech) as described previously (Nishizawa et al., 1999). Genomic Southern blotting was performed according to the manufacturer's instructions. The nucleotide sequence was determined by dideoxy chain termination, using an Applied Biosystems automated sequencer (model 373S) (Nishizawa et al., 1999).

Construction of a gene-disruption plasmid, and integrative conjugation of Microcystis. The plasmids for gene disruption of $m c y F$ by homologous recombination were constructed as follows. The $2.5 \mathrm{~kb} \mathrm{NcoI-EcoRI} \mathrm{fragment} \mathrm{containing} m c y F$ was subcloned from pMCQ3 (Nishizawa et al., 2000) by blunt-end ligation into the SmaI-BamHI site of pUC119 (ToYoBo), generating pCHI621. The $1.2 \mathrm{~kb} \mathrm{XbaI} \mathrm{fragment}$ containing the $\mathrm{Cm}^{\mathrm{R}}$ gene cassette from pR107XH (Nishizawa et al., 1999) and the $1.8 \mathrm{~kb} \mathrm{BamHI}$ fragment containing the mob gene from pSUP5011 (Simon, 1984) were inserted by blunt-end ligation into the BamHI site (in $m c y F$ ) and the HindIII site (in the multi-cloning site) of pCHI621, respectively, generating pJXS10. The plasmids for gene disruption were introduced into Microcystis cells (by conjugation) from E. coli S17-1, and chloramphenicol-resistant $(8 \mu \mathrm{g}$ chloramphenicol ml $\mathrm{m}^{-1}$ ) conjugants were selected as described previously (Nishizawa et al., 1999).

HPLC analysis of microcystins. Microcystins were extracted from the dried cells with $5 \%$ aqueous acetic acid, purified using BondElute ODS cartridges (Varian) and analysed by HPLC as described previously (Nishizawa et al., 1999).

Bacterial complementation test. E. coli WM335, which requires D-glutamic acid for growth, was grown in LuriaBertani (LB) medium supplemented with $50 \mu \mathrm{g}$ D-glutamic acid $\mathrm{ml}^{-1}$ and $20 \mu \mathrm{g}$ thymine $\mathrm{ml}^{-1}$. The racemase expression vector $\mathrm{pQE}-\mathrm{McyF}$ was constructed as follows (see Fig. 4a). The entire $m c y F$ gene was amplified by PCR with primers $5^{\prime}$ $\mathrm{McyF} / S p h \mathrm{I}\left(5^{\prime}\right.$-CT CGCATGCAG ACA AAA CTA CCG-3') and $3^{\prime}-\mathrm{McyF} / \mathrm{HindIII}\left(5^{\prime}-\mathrm{CTC}\right.$ AAGCTT TTT GGG TTT GAA GGC-3') (restriction sites are underlined and modified sequences are shown in italics). PCR was carried out on chromosomal $M$. aeruginosa K-139 DNA with Taq DNA polymerase (Nippon Gene). PCR products were purified, digested with $S p h I$ and HindIII, and cloned into the SphI and HindIII sites of the pQE70 vector (Qiagen). Both the $5^{\prime}$ - and the $3^{\prime}$-cloning sites were confirmed by sequencing. The primers (and sequences) used were as follows: QE/F2 primer, 5'-TTG CTT TGT GAG CGG ATA AC-3'; QE/R1 primer, 5'-CAT TAC TGG ATC TAT CAA CAG G-3'. HindIII digestion of pQE70 resulted in removal of the His-tag sequence and appended the amino acid sequence SLIS at the C-terminus of the recombinant protein.

Expression of McyF. E. coli WM335 cells transformed with pQE-McyF were inoculated into LB medium supplemented with ampicillin $\left(75 \mu \mathrm{g} \mathrm{ml}^{-1}\right)$ and thymine $\left(20 \mu \mathrm{g} \mathrm{ml}^{-1}\right)$ and were grown at $37^{\circ} \mathrm{C}$ with shaking for over $20 \mathrm{~h}$. Expression 
was analysed by SDS-PAGE and staining with Coomassie brilliant blue according to the method of Laemmli (1970).

Computer analysis of DNA. The DNA sequences were assembled and analysed using GENETYX-MAC software (Software Development).

\section{RESULTS AND DISCUSSION}

\section{Characterization of the $\boldsymbol{m c y} \boldsymbol{F}$ gene of $\boldsymbol{M}$. aeruginosa}

Previously, we characterized the microcystin synthetase gene cluster and found the racemase gene, $m c y F$, in this cluster (Nishizawa et al., 2000). The $m c y F$ gene is located $35 \mathrm{bp}$ downstream of the PKS-NRPS hybrid gene $m c y E$, which contains the NRPS module at its $3^{\prime}$ end. The $m c y F$ gene is 753 bp in length, encoding a 251residue polypeptide with a predicted molecular mass of $27990 \mathrm{Da}$. The putative Shine-Dalgarno sequence (AGGAGA) was found before the putative initiation codon. The NRPS module present at the $3^{\prime}$ end of the $m c y E$ gene is presumed to be involved in activation and incorporation of glutamic acid. Moore et al. (1991) demonstrated (by ${ }^{13} \mathrm{C}-\mathrm{NMR}$ analysis) that $\mathrm{D}-\mathrm{Glu}$ in the microcystin molecule is derived from L-Glu, and that DMeAsp does not arise from rearrangement of Glu. In the database search, McyF showed 30.4\% identity with respect to the aspartate racemase of Desulfurococcus (Nishizawa et al., 2000).

D-Glutamic acid is fairly widespread in eubacteria, as Damino acids constitute part of the fundamental tetrapeptide chain of murein in the cell wall (as common components of peptidoglycans) (Fotheringham et al., 1998; Liu et al., 1997; Malathi et al., 1999; Pucci et al., 1995). D-Glutamate in a free form is incorporated into the common peptidoglycan precursor UDP-N-acetylmuramoyl-L-alanyl-D-glutamate (UDP-MurNAc-L-AlaD-Glu), through the action of UDP-MurNAc-L-Ala-DGlu synthetase (EC 6.3.2.9). In bacteria, enzymes are involved in the biosynthesis of D-glutamic acid through two different pathways: D-glutamate racemase and Damino acid aminotransferase (Malathi et al., 1999). Cysteine residues at the active sites of motif 1 $[(\mathrm{V} / \mathrm{I}) \times(\mathrm{P} / \mathrm{A}) \mathrm{CNTA}(\mathrm{H} / \mathrm{T})]$ and motif $2[(\mathrm{I} / \mathrm{V})(\mathrm{A} /$ L) $\operatorname{GCTH}(\mathrm{E} / \mathrm{H}) \times(\mathrm{S} / \mathrm{P})]$, which are thought to form the catalytic centres of these cofactor-independent racemases (Yohda et al., 1996), were also conserved in McyF (Nishizawa et al., 2000).

\section{Disruption of the mcyF gene}

To determine whether the cloned racemase gene, $m c y F$, in the $m c y$ operon is required for microcystin biosynthesis, gene disruption of $m c y F$ in $M$. aeruginosa K139 and Microcystis sp. S-70 was carried out. However, no disruptants were isolated for strain K-139. The restriction barrier of strain K-139 may interfere with recombination (Takahashi et al., 1996). Genomic Southern hybridization demonstrated the presence of $m c y F$ in Microcystis sp. S-70, which produces MCYSTLR, -RR and -YR (Nishizawa et al., 2000). Therefore, insertional mutagenesis of $m c y F$ in strain S-70 was performed, and the chloramphenicol-resistant conjugant Microcystis sp. S-70JX1 was isolated.

To demonstrate integration of the $\mathrm{Cm}^{\mathrm{R}}$ cassette into the $m c y F$ gene on the chromosome, genomic Southern hybridization was carried out. Total DNA from mutant S-70JX1 was digested with either HincII or HindIII and then probed with the $0.75 \mathrm{~kb} \mathrm{BglII}$ fragment containing a part of the racemase gene (Fig. 2a). A $2 \cdot 9 \mathrm{~kb}$ signal and a $5.0 \mathrm{~kb}$ signal, obtained with HincII and HindIII, respectively, were detected in the control (Microcystis sp. S-70). A $0.8 \mathrm{~kb}$ signal and a $6.2 \mathrm{~kb}$ signal, however, were detected in mutant JX1 (Fig. 2b). In the case of HindIII digestion, the position of this signal coincided with that of a signal obtained with the probe of the $\mathrm{Cm}^{\mathrm{R}}$ cassette fragment (data not shown). Furthermore, to confirm $m c y F$ gene disruption and gene organization in (a)

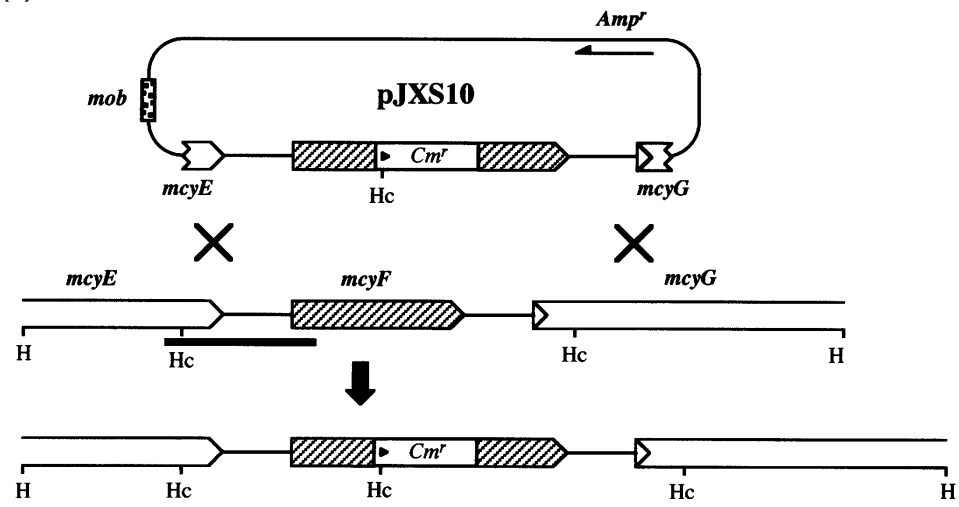

(b)

$\begin{array}{llll}1 & 2 & 3 & 4\end{array}$

Microcystis sp. S-70

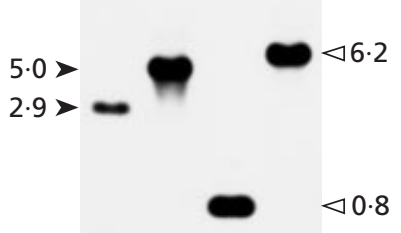

JX1 mutants

Fig. 2. Southern blot analysis of the mcyF-disrupted conjugant. (a) Schematic representation of insertional inactivation of the mcyF gene. The mcyF probe (black bar) is shown. Hc, Hincll; $\mathrm{H}$, Hindlll. (b) Genomic Southern hybridization analysis. Chromosomal DNA of Microcystis sp. S-70 was digested with either Hincll or Hindlll. Lanes: 1, wild-type with Hincll; 2, wild-type with HindIII; 3, JX1 mutant with Hincll; 4, JX1 mutant with HindIII. The sizes of the signals are indicated in kb on both sides. 


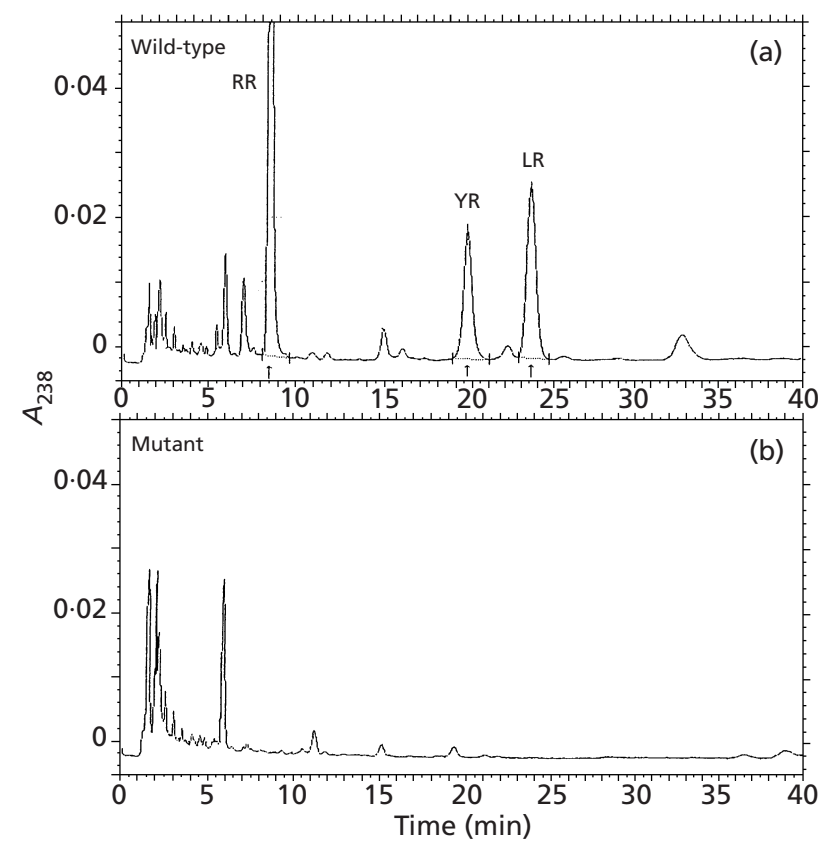

Fig. 3. HPLC of methanol extracts of Microcystis sp. S-70 cells Microcystins were extracted from dried cells of the wild-type (a) and the mutant JX1 (b). Peaks: RR, MCYST-RR; YR, MCYST-YR; LR, MCYST-LR. Column, Cosmosil 5C18ARII $(150 \times 4.6 \mathrm{~mm}$ i.d.); mobile phase, $\mathrm{CH}_{3} \mathrm{CN} / 0.01 \mathrm{M}$ trifluoroacetic acid $(30: 70, \mathrm{v} / \mathrm{v})$; flow rate, $1.0 \mathrm{ml} \mathrm{min}^{-1}$; detection, $238 \mathrm{~nm}$.

strain S-70JX1, the $6.2 \mathrm{~kb}$ HindIII fragment, including $m c y E-m c y F-m c y G$ (Fig. 2b), was cloned from this strain. Sequence analysis showed that the gene organization in strain S-70 was the same as that in strain $\mathrm{K}-139$, and nucleotide sequence identity between both strains was $95 \cdot 5 \%$.

To investigate the role of $m c y F$, we used HPLC to analyse microcystin production. The extracts from the control (Microcystis sp. S-70) showed three peaks for MCYST-LR, -RR and -YR (Fig. 3a). In contrast, the S$70 \mathrm{JX} 1$ mutant lacked all of these microcystin peaks (Fig. $3 \mathrm{~b})$. Moreover, the $m c y F$ mutants showed normal growth and development on agarose plates and in liquid culture, indicating that McyF has no essential housekeeping function. These observations revealed that the $m c y F$ gene encoding racemase is specifically involved in microcystin biosynthesis.

Genomic Southern hybridization using a $m c y F$ probe revealed the absence of $m c y F$ in microcystin-nonproducing Microcystis strains and in Synechocystis PCC 6803 (Nishizawa et al., 2000). Two racemase genes, Dglutamate racemase and $\mathrm{D}$-alanine racemase, are found in microcystin-non-producing Synechocystis PCC 6803 in the database of the Kazusa Institute, Chiba, Japan. The $m c y F$-disruptant exhibited significant growth similar to that of the control strain. Therefore, the $m c y F$ gene is required only for microcystin synthesis, but not for fundamental growth.
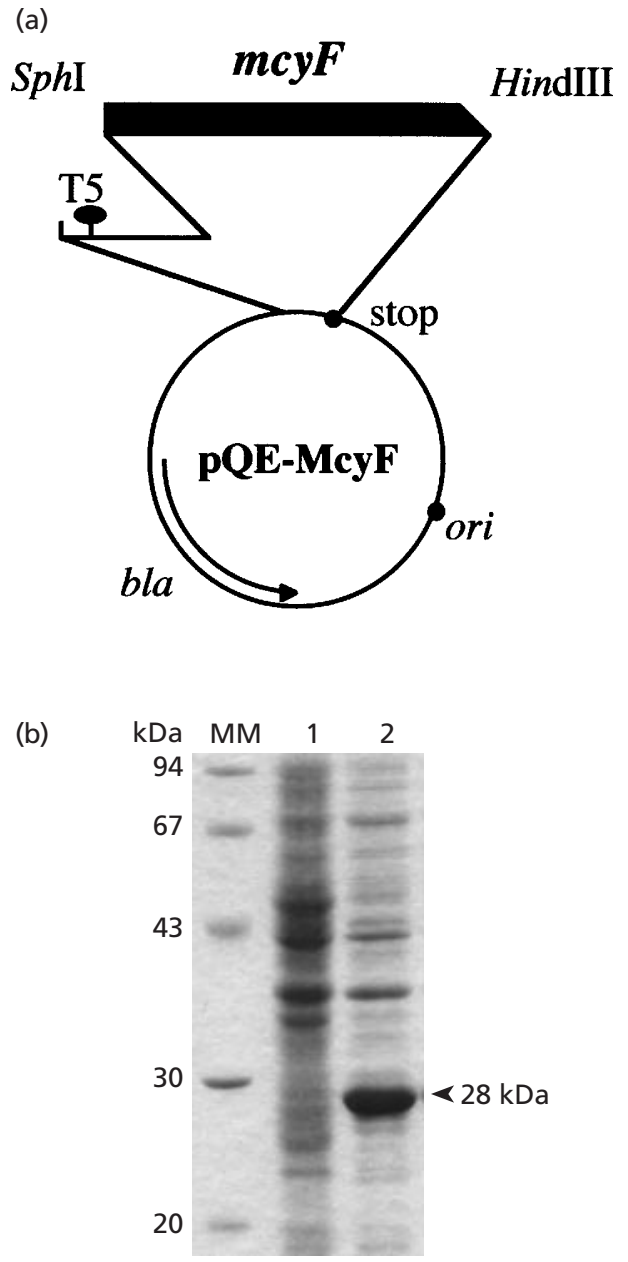

Fig. 4. Overexpression of the recombinant McyF protein for complementation of an $E$. coli glutamate racemase mutant. (a) Recombinant plasmid pQE-McyF. T5, promoter; stop, stop codon. (b) SDS-PAGE analysis of McyF expressed from pQE-McyF in E. coli WM335. A Coomassie-stained gel of the total cell extracts is shown. Lanes: 1, pQE70; 2, pQE-McyF; MM, molecular mass markers $(\mathrm{kDa})$. Arrowhead, position of the $28 \mathrm{kDa}$ expressed McyF protein.

\section{Complementation of a bacterial mutant deficient in D-Glu synthesis}

To confirm that the $m c y F$ gene encodes the glutamate racemase, the McyF racemase was expressed in $\mathrm{D}$ glutamic-acid-requiring E. coli WM335, using the T5 promoter transcription-translation system of $\mathrm{pQE}$ vectors (Fig. 4a). E. coli WM335 transformed with pQEMcyF expressed a protein of about $28 \mathrm{kDa}$ (Fig. 4b), the molecular mass of which coincided well with the value predicted for McyF on the basis of its nucleotide sequence. The pQE-McyF-transformed E. coli grew exponentially and survived on agarose plates and in LB liquid medium (data not shown) without D-glutamic acid as well as in those supplemented with $50 \mu \mathrm{g}$ Dglutamic acid $\mathrm{ml}^{-1}$. However, E. coli WM335 carrying the expression vector pQE70 or pCHI621 showed no 


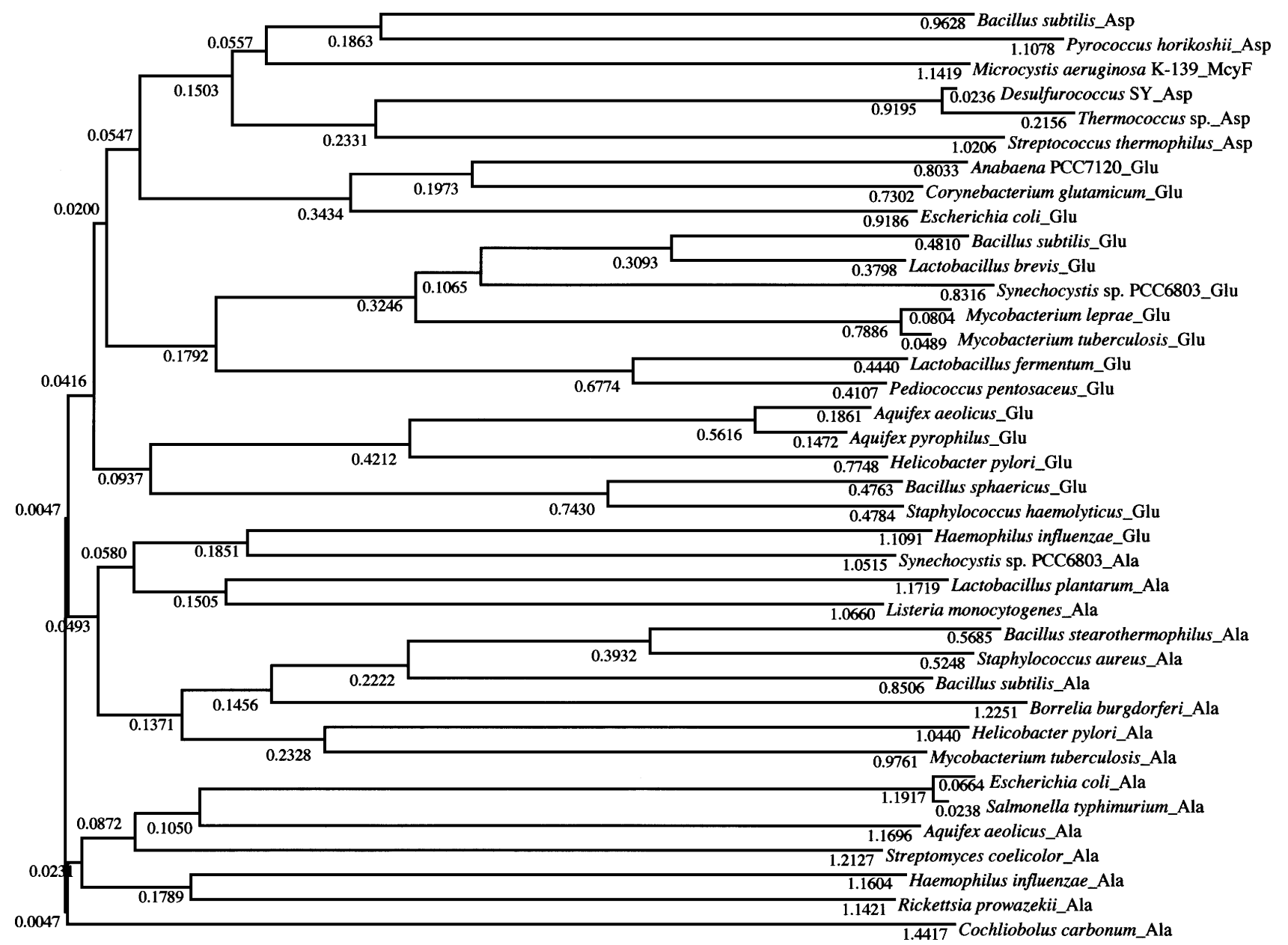

Fig. 5. Phylogenetic tree representation of the apparent evolutionary distances of amino acid racemase proteins from several bacteria and fungi. The tree was constructed by the neighbour-joining method. The scale for the tree indicates inferred evolutionary distances. Anabaena PCC 7120_Ala, AAF33755; Aquifex aeolicus_Ala, O67687; Aquifex aeolicus_Glu, O66662; Aquifex pyrophilus_Glu, P56868; Bacillus sphaericus_Glu, P52972; Bacillus stearothermophilus_Ala, P10724; Bacillus subtilis_Ala, P94494; Bacillus subtilis_Asp, Z99118; Bacillus subtilis_Glu, P94556; Borrelia burgdorferi_Ala, O51182; Cochliobolus carbonum_Ala, AF169478; Corynebacterium glutamicum_Glu, AB020624; Desulfurococcus SY_Asp, D84067; Escherichia coli_Ala, AAC43147; Escherichia coli_Glu, AE000471; Haemophilus influenzae_Ala, P45257; Haemophilus influenzae_Glu, P52973; Helicobacter pylori_Ala, O25595; Helicobacter pylori_Glu, P56068; Lactobacillus brevis_Glu, P48797; Lactobacillus fermentum_Glu, Q03469; Lactobacillus plantarum_Ala, O08445; Listeria monocytogenes_Ala, O85045; Microcystis aeruginosa K-139, AB032549; Mycobacterium leprae_Glu, P46705; Mycobacterium tuberculosis_Ala, Q50705; Mycobacterium tuberculosis_Glu, Q10626; Pediococcus pentosaceus_Glu, Q08783; Pyrococcus horikoshii_Asp, AP00003; Rickettsia prowazekii_Ala, Q9ZE52; Salmonella typhimurium_Ala, P06655; Staphylococcus aureus_Ala, Q9ZAH5; Staphylococcus haemolyticus_Glu, P52974; Streptococcus thermophilus_Asp, X61301; Streptomyces coelicolor_Ala, O86786; Synechocystis sp. PCC 6803_Ala, S75775; Synechocystis sp. PCC 6803_Glu, P73737; Thermococcus sp._Asp, BAA35091.

growth on agarose plates without D-glutamic acid, and a rapid decrease in $\mathrm{OD}_{660}$ was observed shortly after inoculation into liquid medium without D-glutamic acid (data not shown). These observations showed that the $m c y F$ gene was capable of complementation of the Dglutamic-acid-requiring phenotype in E. coli. Gene disruption and complementation analysis demonstrated that D-Glu in the cyclic heptapeptide microcystin is synthesized from the primary metabolite L-Glu by a glutamate racemase $(\mathrm{McyF})$. These results indicated that McyF is involved in D-glutamic acid synthesis. Moreover, this is the first report showing the occurrence of a glutamate racemase gene in prokaryotic nonribosomal peptide synthesis.

\section{Phylogenetic analysis of the D-amino acid racemase}

The microcystin molecule is composed of the following three D-amino acid residues: D-Ala, D-MeAsp and D-Glu (Fig. 1). The microcystin synthetase was shown previously to contain one epimerization domain in McyA and one racemase, McyF (Nishizawa et al., 2000). In NRPS, D-amino acid synthesis is generally catalysed by an epimerase, which is encoded by an epimerization 
domain at the carboxy-terminal end of the thiolation domain (Stein et al., 1995). Our results indicated that a glutamic acid racemase, McyF, is responsible for the production of D-Glu.

On the other hand, a sequence similarity search of Damino acid racemases from bacteria and fungi, which are available in the GenBank, EMBL and DDBJ databases, was carried out using the program in GENETYXMAC (see Methods). Our results revealed that McyF has $22 \cdot 4-30 \cdot 1 \%$ sequence similarity to aspartate racemase, $16 \cdot 9-23.7 \%$ similarity to glutamate racemase, and $15 \cdot 1-$ $21 \cdot 2 \%$ similarity to alanine racemase. The phylogenetic relationships between these racemase genes were analysed by the neighbour-joining method, using GENETYXMAC, and the resulting dendrogram is shown in Fig. 5. These racemase genes were tightly clustered, except for the glutamate racemase of Haemophilus influenzae. DAmino acid racemases can be roughly divided into two groups, namely the Ala type and the Glu type. Aspartate racemases belong to the glutamate racemase cluster. Two cysteine residues, which are thought to form the catalytic centre, were highly conserved among the aspartate and glutamate racemases, as well as among their surrounding amino acid sequences (Nishizawa et al., 2000).

The only racemase previously reported to be involved in non-ribosomal peptide synthesis was the alanine racemase in fungi (Cheng \& Walton, 2000; Hoffmann et al., 1994). The involvement of specific racemases in prokaryotic non-ribosomal peptide synthesis has not been reported. Their racemase genes are unrelated, at the primary amino acid level, to any known bacterial alanine racemases (Cheng \& Walton, 2000; Hoffmann et al., 1994). This analysis indicated that McyF belongs to the glutamic acid racemase group. Microcystin synthetase, including both types of D-amino acid synthesis enzymes, epimerase and racemase, is a unique NRPS. Interestingly, McyF is grouped with aspartate racemases in the phylogenetic tree (Fig. 5). Since the microcystin molecule includes $\mathrm{D}-\mathrm{MeAsp}$, it is possible that McyF is responsible for D-MeAsp synthesis together with D-Glu synthesis. However, this possibility has been excluded by the work of Moore et al. (1991), who concluded from the $\left[1,2-{ }^{13} \mathrm{C}\right]$ acetate labelling pattern that MeAsp is synthesized from acetyl-CoA and pyruvic acid.

\section{ACKNOWLEDGEMENTS}

We thank Drs K.-I. Harada and K. Fujii for their help with HPLC analysis. We are grateful to Drs W. Messer and M. Wachi for gifts of the strains used in this study.

\section{REFERENCES}

Carmichael, W. W. (1994). The toxins of cyanobacteria. Sci Am 270, 64-72.

Cheng, Y. \& Walton, J. D. (2000). A eukaryotic alanine racemase gene involved in cyclic peptide biosynthesis. J Biol Chem 275, 4906-4911.

Fotheringham, I. G., Bledig, S. A. \& Taylor, P. P. (1998). Charac- terization of the genes encoding D-amino acid transaminase and glutamate racemase, two D-glutamate biosynthetic enzymes of Bacillus sphaericus ATCC 10208. J Bacteriol 180, 4319-4323.

Harada, K., Ogawa, K., Matsuura, K. \& 7 other authors (1991). Isolation of two toxic heptapeptide microcystins from an axenic strain of Microcystis aeruginosa K-139. Toxicon 29, 479-489.

Hoffmann, K., Scheider-Scherzer, E., Kleinkauf, H. \& Zocher, R. (1994). Purification and characterization of eucaryotic alanine racemase acting as key enzyme in cyclosporin biosynthesis. J Biol Chem 269, 12710-12714.

Jochimsen, E. M., Carmichael, W. W., An, J. S. \& 9 other authors (1998). Liver failure and death after exposure to microcystins at a hemodialysis center in Brazil. N Engl J Med 338, 873-878.

Laemmli, U. K. (1970). Cleavage of structural proteins during the assembly of the head of bacteriophage T4. Nature 227, 680-685.

Liu, L., Yoshimura, T., Endo, K., Esaki, N. \& Soda, K. (1997). Cloning and expression of the glutamate racemase gene of Bacillus pumilus. J Biochem 121, 1156-1161.

Lugtenberg, E. J. J., Wijsman, H. J. W. \& van Zaane, D. (1973). Properties of a D-glutamic acid-requiring mutant of Escherichia coli. J Bacteriol 114, 499-506.

MacKintosh, C., Beatte, K. A., Klumpp, S., Cohen, P. \& Codd, G. A. (1990). Cyanobacterial microcystin-LR is a potent and specific inhibitor of protein phosphatases 1 and 2A from both mammals and higher plants. FEBS Lett 264, 187-192.

Malathi, K. C., Wachi, M. \& Nagai, K. (1999). Isolation of the murI gene from Brevibacterium lactofermentum ATCC 13869 encoding D-glutamate racemase. FEMS Microbiol Lett 175, 193-196.

Marahiel, M. A. (1997). Protein templates for the biosynthesis of peptide antibiotics. Chem Biol 4, 561-567.

Moore, R. E., Chen, J. L., Moore, B. S. \& Patterson, G. M. L. (1991). Biosynthesis of Microcystin-LR. Origin of the carbons in the Adda and Masp units. J Am Chem Soc 113, 5083-5084.

Nishizawa, T., Asayasma, M., Fujii, K., Harada, K.-I. \& Shirai, M. (1999). Genetic analysis of the peptide synthetase genes for a cyclic heptapeptide microcystin in Microcystis spp. J Biochem 126, 520-529.

Nishizawa, T., Ueda, A., Asayasma, M., Fujii, K., Harada, K.-I., Ochi, K. \& Shirai, M. (2000). Polyketide synthase gene coupled to the peptide synthetase module involved in the biosynthesis of the cyclic heptapeptide microcystin. J Biochem 127, 779-789.

Pucci, M. J., Thanassi, J. A., Ho, H., Falk, P. J. \& Dougherty, T. J. (1995). Staphylococcus haemolyticus contains two D-glutamic acid biosynthetic activities, a glutamate racemase and a D-amino acid transaminase. J Bacteriol 177, 336-342.

Sakamoto, T., Shirai, M., Asayama, M., Aida, T., Sato, A., Tanaka, K., Takahashi, H. \& Nakano, M. (1993). Characteristics of DNA and multiple rpoD homologs of Microcystis (Synechocystis) strains. Int J Syst Bacteriol 43, 844-847.

Sambrook, J., Fritsch, E. F. \& Maniatis, T. (1989). Molecular Cloning: a Laboratory Manual, 2nd edn. Cold Spring Harbor, NY : Cold Spring Harbor Laboratory.

Shirai, M., Ohtake, A., Sano, T. \& 8 other authors (1991). Toxicity and toxins of natural blooms and isolated Microcystis spp. (Cyanobacteria) and improved procedure for purification of cultures. Appl Environ Microbiol 57, 1241-1245.

Simon, R. (1984). High frequency mobilization of gram-negative bacterial replicons by the in vitro constructed Tn5-Mob transposon. Mol Gen Genet 196, 413-420.

Sivonen, K. \& Jones, G. (1999). Cyanobacterial toxins. In Toxic Cyanobacteria in Water: a Guide to their Public Health Conse- 
quences, Monitoring and Management, pp. 41-111. Edited by I. Chorus \& J. Bertram. London: E. \& F. N. Spon.

Stachelhaus, T. \& Marahiel, M. A. (1995). Modular structure of genes encoding multifunctional peptide synthetases required for non-ribosomal peptide synthesis. FEMS Microbiol Lett 125, 3-14.

Stein, T., Kluge, B., Vater, J., Franke, P., Otto, A. \& WittmannLiebold, B. (1995). Gramicidin S synthetase 1 (phenylalanine racemase), a prototype of amino acid racemases containing the cofactor 4'-phosphopantetheine. Biochemistry 34, 4633-4642.

Takahashi, I., Hayano, D., Asayama, M., Futagami, M., Watahiki, M. \& Shirai, M. (1996). Restriction barrier composed of an extracellular nuclease and restriction endonuclease in the uni- cellular cyanobacterium Microcystis sp. FEMS Microbiol Lett 145, 107-111.

Watanabe, M. F., Harada, K., Carmichael, W. W. \& Fujiki, H. (editors) (1996). Toxic Microcystis. Boca Raton, FL: CRC Press. Yohda, M., Endo, I., Abe, Y., Ohta, T., lida, T., Maruyama, T. \& Kagawa, Y. (1996). Gene for aspartate racemase from the sulfurdependent hyperthermophilic archaeum, Desulfurococcus strain SY. J Biol Chem 271, 22017-22021.

Received 3 October 2000; revised 6 December 2000; accepted 29 January 2001. 